\title{
Four fermion operators and the search for BSM Physics
}

\author{
Simon Catterall ${ }^{* \dagger}$ \\ Physics Department, Syracuse University \\ E-mail: smcephysics.syr.edu
}

\section{Aarti Veernala}

Physics Department, Syracuse University

E-mail: aveernalesyr.edu

\begin{abstract}
We report on Monte Carlo simulations focused on elucidating the phase structure of a $S U(2)$ gauge theory containing $N_{f}$ Dirac fermion flavors transforming in the fundamental representation of the group and interacting through an additional chirally invariant four fermion term. Pairs of physical flavors are implemented using the two tastes present in a reduced staggered fermion formulation of the theory with the Yukawa interactions necessary for generating the four fermion term preserving the usual shift symmetries. We observe a crossover in the behavior of the chiral condensate for strong four fermi coupling associated with the generation of a dynamical mass for the fermions. At weak gauge coupling this crossover is consistent with the usual continuous phase transition seen in the pure (ungauged) NJL model. However, if the gauge coupling is strong enough to cause confinement we observe a much more rapid crossover in the chiral condensate consistent with a first order phase transition
\end{abstract}

The 30 International Symposium on Lattice Field Theory - Lattice 2012,

June 24-29, 2012

Cairns, Australia

\footnotetext{
* Speaker.

${ }^{\dagger}$ Work supported in part by DOE grant DE-FG02-85ER40237
} 


\section{Introduction}

Elucidating the nature of the electroweak symmetry breaking sector of the Standard Model (SM) is the main goal of the Large Hadron Collider currently running at CERN. It is widely believed that the simplest scenario involving a single scalar Higgs field is untenable due to the fine tuning and triviality problems which arise in scalar field theories. One natural solution to these problems can be found by assuming that the Higgs sector in the Standard Model arises as an effective field theory describing the dynamics of a composite field arising from strongly bound fermion-antifermion pairs. These models are generically called technicolor theories.

However, to obtain fermion masses in these scenarios requires additional model building, as in extended technicolor models $[1,2,3,4]$ and models of top-condensation $[5,6,7,8]$. In the latter models four-fermion interactions drive the formation and condensation of a scalar top-anti-top bound state which plays the role of the Higgs at low energies.

Our motivation in this paper is to study how the inclusion of such four fermion interactions may influence the phase structure and low energy behavior of non-abelian gauge theories in general. Specifically we have examined a model with both gauge interactions and a chirally invariant four fermi interaction - a model known in the literature as the gauged NJL model [9].

The focus of the current work is to explore the phase diagram when fermions are charged under a non-abelian gauge group. Indeed, arguments have been given in the continuum that the gauged NJL model may exhibit different critical behavior at the boundary between the symmetric and broken phases ${ }^{1}$ corresponding to the appearance of a line of new fixed points associated with a mass anomalous dimension varying in the range $1<\gamma_{\mu}<2[9,10]$. The evidence for this behavior derives from calculations utilizing the ladder approximation in Landau gauge to the SchwingerDyson equations. A primary goal of the current study was to use lattice simulation to check the validity of these conclusions and specifically to search for qualitatively new critical behavior in the gauged model as compared to the pure NJL theory. While we will present results that indicate that the phase structure of the gauged NJL model is indeed different from pure NJL, we shall argue that our results are not consistent with the presence of any new fixed points in the theory.

In the work reported here and described in detail in [11] we have concentrated on the four flavor theory corresponding to two copies of the basic Dirac doublet used in the lattice construction. The four flavor theory is expected to be chirally broken and confining at zero four fermi coupling and is free from sign problems for gauge group $S U(2)$. Understanding the effects of the four fermion term in this theory can then serve as a benchmark for future studies of theories which, for zero four fermi coupling, lie near or inside the conformal window. In the latter case the addition of a four fermion term will break conformal invariance but in principle that breaking may be made arbitrarily small by tuning the four fermi coupling. It is entirely possible that the phase diagrams of such conformal or walking theories in the presence of four fermi terms may exhibit very different features than those seen for a confining gauge theory.

\footnotetext{
${ }^{1}$ Notice that the appearance of a true phase transition in the gauged NJL models depends on the approximation that we can neglect the running of the gauge coupling
} 


\section{Details of the model}

We will consider a model which consists of $N_{f} / 2$ doublets of gauged massless Dirac fermions in the fundamental representation of an $S U(2)$ gauge group and incorporating an $S U(2)_{L} \times S U(2)_{R}$ chirally invariant four fermi interaction. The action for a single doublet takes the form

$$
\begin{aligned}
S & =\int d^{4} x \bar{\psi}(i \not \partial-\not A) \psi-\frac{G^{2}}{2 N_{f}}\left[(\bar{\psi} \psi)^{2}+\left(\bar{\psi} i \gamma_{5} \tau^{a} \psi\right)^{2}\right] \\
& -\frac{1}{2 g^{2}} \operatorname{Tr}\left[F_{\mu \nu} F^{\mu v}\right],
\end{aligned}
$$

where $\mathrm{G}$ is the four-fermi coupling, $g$ the usual gauge coupling and $\tau^{a}, a=1 \ldots 3$ are the generators of the $S U(2)$ flavour group.

This action may be discretized using the (reduced) staggered fermion formalism with the result

$$
S=\sum_{x, \mu} \chi^{T}(x) \mathscr{U}_{\mu}(x) \chi\left(x+a_{\mu}\right)\left[\eta_{\mu}(x)+G \bar{\phi}_{\mu}(x) \varepsilon(x) \xi_{\mu}(x)\right]
$$

where $\eta_{\mu}(x), \xi_{\mu}(x)$ and $\varepsilon(x)$ are the usual staggered fermion phases, $\bar{\phi}(x)=\frac{1}{16} \sum_{h} \phi(x-h)$ the average of the scalar field over the hypercube $[12,13]$ and the gauge field acting on the reduced staggered fermions takes the form:

$$
\mathscr{U}_{\mu}(x)=\frac{1}{2}[1+\varepsilon(x)] U_{\mu}(x)+\frac{1}{2}[1-\varepsilon(x)] U_{\mu}^{*}(x) .
$$

Clearly the theory is invariant under the $U(1)$ symmetry $\chi(x) \rightarrow e^{i \alpha \varepsilon(x)} \chi(x)$ which is to be interpreted as the $U(1)$ symmetry corresponding to fermion number. More interestingly it is also invariant under certain shift symmetries given by

$$
\begin{aligned}
\chi(x) & \rightarrow \xi_{\rho}(x) \chi(x+\rho), \\
U_{\mu}(x) & \rightarrow U_{\mu}^{*}(x+\rho), \\
\phi_{\mu}(x) & \rightarrow(-1)^{\delta_{\mu \rho}} \phi_{\mu}(x+\rho) .
\end{aligned}
$$

These shift symmetries correspond to a discrete subgroup of the continuum axial flavor transformations which act on the matrix field $\Psi$ according to

$$
\Psi \rightarrow \gamma_{5} \Psi \gamma_{\rho}
$$

Notice that no single site mass term is allowed in this model.

\section{Numerical results}

We have used the RHMC algorithm to simulate the lattice theory with a standard Wilson gauge action being employed for the gauge fields. Upon integration over the basic fermion doublet we obtain a Pfaffian $\operatorname{Pf}(\mathrm{M}(\mathrm{U}))$ depending on the gauge field ${ }^{2}$. The required pseudofermion weight for $N_{f}$ flavors is then $\operatorname{Pf}(M)^{N_{f} / 2}$. The pseudoreal character of $S U(2)$ allows us to show that the Pfaffian

\footnotetext{
${ }^{2}$ Note that the fermion operator appearing in eqn. 2.2 is antisymmetric
} 
is purely real and so we are guaranteed to have no sign problem if we use multiples of four flavors corresponding to a pseudofermion operator of the form $\left(M^{\dagger} M\right)^{-\frac{N_{f}}{8}}$. The results in this paper are devoted to the case $N_{f}=4$. We have utilized a variety of lattice sizes: $4^{4}, 6^{4}, 8^{4}$ and $8^{3} \times 16$ and a range of gauge couplings $1.8<\beta \equiv 4 / g^{2}<10.0$. To determine where the pure gauge theory is

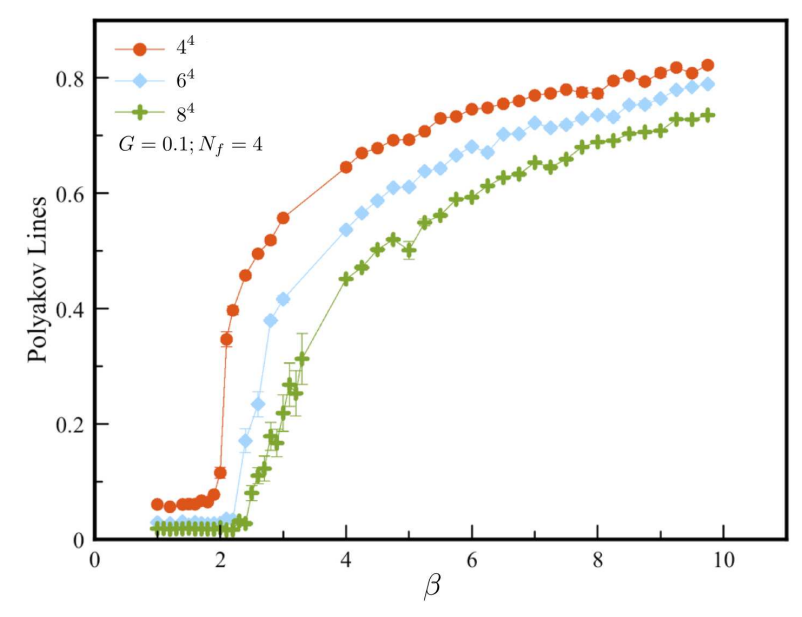

Figure 1: Polyakov loop vs $\beta$ at $G=0.1$ for four flavours

strongly coupled and confining we have examined the average Polyakov line as $\beta$ varies holding the four fermi coupling fixed at $G=0.1$. This is shown in figure 1 . We see a strong crossover between a confining regime for small $\beta$ to a deconfined regime at large $\beta$. The crossover coupling is volume dependent and takes the value of $\beta_{c} \sim 2.4$ for lattices of size $L=8$. For $\beta<1.8$ the plaquette drops below 0.5 which we take as indicative of the presence of strong lattice spacing artifacts and so we have confined our simulations to larger values of $\beta$. We have set the fermion mass to zero in all of our work so that our lattice action possesses the series of exact chiral symmetries discussed earlier.

One of the primary observables used in this study is the chiral condensate which is computed from the gauge invariant one link mass operator

$$
\chi(x)\left(\mathscr{U}_{\mu}(x) \chi\left(x+e_{\mu}\right)+\mathscr{U}_{\mu}^{\dagger}\left(x-e_{\mu}\right) \chi\left(x-e_{\mu}\right)\right) \varepsilon(x) \xi_{\mu}(x)
$$

In Figure 2 we show a plot of the absolute value of the condensate at a variety of gauge couplings $\beta$ on $8^{4}$ lattices. Notice the rather smooth transition between symmetric and broken phases around $G \sim 0.9$ for $\beta=10$. This is consistent with earlier work using sixteen flavors of naive fermion reported in [14] which identified a line of second order phase transitions in this region of parameter space. It also agrees with the behavior seen in previous simulations using conventional staggered quarks [15].

This behavior should be contrasted with the behavior of the condensate for strong gauge coupling $\beta \leq 2.4$. Here a very sharp transition can be seen reminiscent of a first order phase transition. In Figure 3 we highlight this by showing a plot of the condensate versus four fermi coupling at the single gauge coupling $\beta=2.0$ for a range of different lattice sizes. The chiral condensate is 


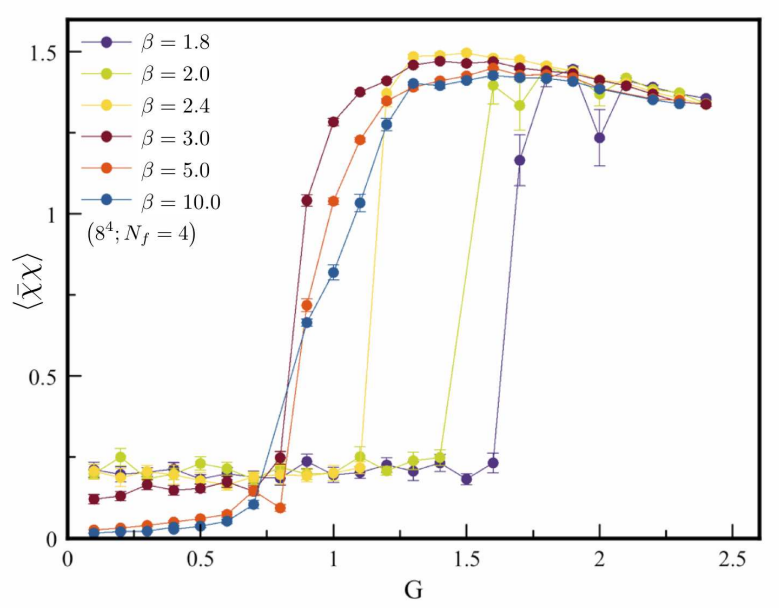

Figure 2: $\langle\bar{\chi} \chi\rangle$ vs $G$ for varying $\beta$ for the $8^{4}$ lattice with $N_{f}=4$.

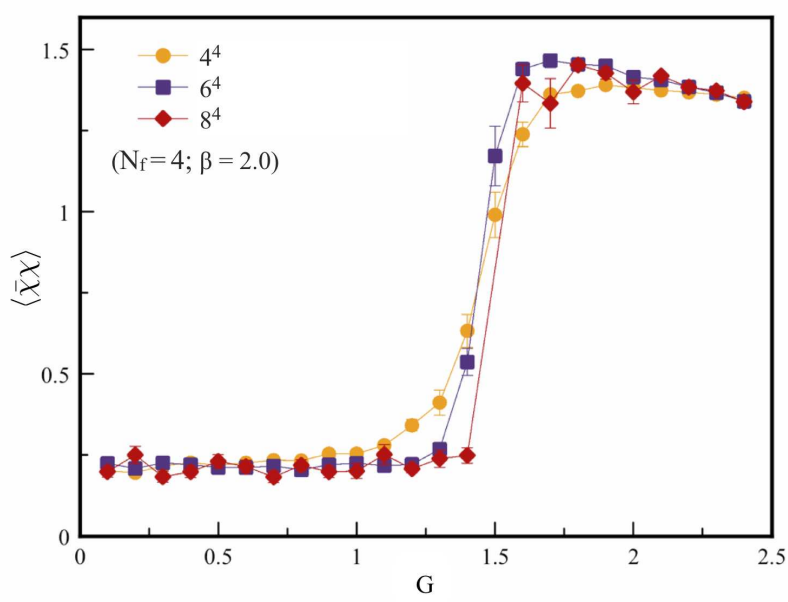

Figure 3: $\langle\bar{\chi} \chi\rangle$ vs $G$ at $\beta=2.0$ for lattices $4^{4}, 6^{4}$ and $8^{4}$ with $N_{f}=4$.

now non-zero even for small four fermi coupling and shows no strong dependence on the volume consistent with spontaneous chiral symmetry breaking in the pure gauge theory. However, it jumps abruptly to much larger values when the four fermi coupling exceeds some critical value. This crossover or transition is markedly discontinuous in character - reminiscent of a first order phase transition. Indeed, while the position of the phase transition is only weakly volume dependent it appears to get sharper with increasing volume.

What seems clear is that the second order transition seen in the pure NJL model is no longer present when the gauge coupling is strong. In the next section we will argue that this is to be expected - in the gauged model one can no longer send the fermion mass to zero by adjusting the 
four fermi coupling since it receives a contribution from gauge mediated chiral symmetry breaking. Indeed the measured one link chiral condensate operator is not an order parameter for such a transition since we observe it to be non-zero for all $G$. Notice however that we see no sign that this condensate depends on the gauge coupling $\beta$ in the confining regime at small $G$. This is qualitatively different from the behavior of regular staggered quarks and we attribute it to the fact that the reduced formalism does not allow for a single site mass term or an exact continuous chiral symmetry. Thus the spontaneous breaking of the residual discrete lattice chiral symmetry by gauge interactions will not be signaled by a light Goldstone pion and the measured condensate will receive contributions only from massive states. The transition we observe is probably best thought of as a crossover phenomenon corresponding to the sudden onset of a new mechanism for dynamical mass generation due to the strong four fermi interactions.

\section{Summary}

In this paper we have conducted numerical simulations of the gauged NJL model for four flavors of Dirac fermion in the fundamental representation of the $S U(2)$ gauge group. We have employed a reduced staggered fermion discretization scheme which allows us to maintain an exact subgroup of the continuum chiral symmetries.

We have examined the model for a variety of values for lattices size, gauge coupling, and four fermi interaction strength. In the NJL limit $\beta \rightarrow \infty$ we find evidence for a continuous phase transition for $G \sim 1$ corresponding to the expected spontaneous breaking of chiral symmetry. However, for gauge couplings that generate a non-zero chiral condensate even for $G=0$ this transition or crossover appears much sharper and there is no evidence of critical fluctuations in the chiral condensate.

Thus our results are consistent with the idea that the second order phase transition which exists in the pure NJL theory $(\beta=\infty)$ survives at weak gauge coupling. However our results indicate that any continuous transition ends if the gauge coupling becomes strong enough to cause confinement. In this case we do however see evidence of additional dynamical mass generation for sufficiently large four fermi coupling associated with an observed rapid crossover in the chiral condensate and a possible first order phase transition.

The fact that we find the condensate non-zero and constant for strong gauge coupling and $G<G_{c}$ shows that the chiral symmetry of the theory is already broken as expected for $S U(2)$ with $N_{f}=4$ flavors. This breaking of chiral symmetry due to the gauge interactions is accompanied by the generation of a non-zero fermion mass even for small four fermi coupling. Notice that this type of scenario is actually true of top quark condensate models in which the strong QCD interactions are already expected to break chiral symmetry independent of a four fermion top quark operator. The magnitude of this residual fermion mass is not controlled by the four fermi coupling and cannot to sent to zero by tuning the four fermi coupling - there can be no continuous phase transition in the system as we increase the four fermi coupling - rather the condensate becomes strongly enhanced for large $G$. 


\section{Acknowledgments}

The simulations were carried out using USQCD resources at Fermilab and Jlab.

\section{References}

[1] E. Eichten and K. Lane, "Dynamical breaking of weak interaction symmetries," Phys.Lett.B, vol. 90, no. $1-2$, pp. $125-130,1980$.

[2] S. Dimopoulos and L. Susskind, "Mass without scalars," Nuclear Physics B, vol. 155, no. 1, pp. 237 $252,1979$.

[3] N. D. Christensen and R. Shrock, "Extended technicolor models with two ETC groups," Phys.Rev., vol. D74, p. 015004, 2006.

[4] T. Appelquist, M. Piai, and R. Shrock, "Fermion masses and mixing in extended technicolor models," Phys.Rev., vol. D69, p. 015002, 2004.

[5] V. Miransky, M. Tanabashi, and K. Yamawaki, "Dynamical Electroweak Symmetry Breaking with Large Anomalous Dimension and t Quark Condensate,” Phys.Lett., vol. B221, p. 177, 1989.

[6] V. Miransky, M. Tanabashi, and K. Yamawaki, "Is the t Quark Responsible for the Mass of W and Z Bosons?," Mod.Phys.Lett., vol. A4, p. 1043, 1989.

[7] W. A. Bardeen, C. T. Hill, and M. Lindner, "Minimal Dynamical Symmetry Breaking of the Standard Model," Phys.Rev., vol. D41, p. 1647, 1990.

[8] W. J. Marciano, "Dynamical Symmetry Breaking and the Top Quark Mass,” Phys.Rev., vol. D41, p. 219, 1990.

[9] K. Yamawaki, "Dynamical symmetry breaking with large anomalous dimension,” 1996. arXiv:hep-ph/9603293v1.

[10] H. S. Fukano and F. Sannino, "Conformal window of gauge theories with four-fermion interactions and ideal walking technicolor,” Phys. Rev. D, vol. 82, p. 035021, Aug 2010.

[11] S. Catterall, J. Hubisz, R. Galvez, D. Mehta and A. Veernala, "Gauged non-abelian NJL models on the lattice", Phys. Rev. D86 2012, 034502.

[12] C. V. den Doel and J. Smit, "Dynamical symmetry breaking in two flavor su(n) and so(n) lattice gauge theories," Nuclear Physics B, vol. 228, no. 1, pp. 122 - 144, 1983.

[13] W. Bock, J. Smit, and J. C. Vink, "Fermion-higgs model with reduced staggered fermions," Phys.Lett.B, vol. 291, p. 297, 1992.

[14] A. Hasenfratz, "The equivalence of the top quark condensate and the elementary higgs field," Nuc. Phys. B., vol. 365, pp. 79-97, Apr 1991.

[15] S. Hands and J. B. Kogut, "Logarithmic corrections to the equation of state in the SU(2) x SU(2) Nambu-Jona-Lasinio model,” Nucl.Phys., vol. B520, pp. 382-408, 1998. 